\title{
Dengue Vector Control in Malaysia- Challenges and Recent Advances
}

\author{
Lee HL, Rohani A, Khadri MS, Nazni WA, Rozilawati H, Nurulhusna AH, Nor Afizah AH, Roziah A, Rosilawati R, \\ Teh $\mathrm{CH}$.
}

Medical Entomology Unit \& WHO Collaborating Centre for the Ecology, Taxonomy and Control of Vectors of Malaria, Filariasis \& Dengue, Institute for Medical Research, Jalan Pahang, 50588 Kuala Lumpur, Malaysia.

\begin{abstract}
Dengue is a serious mosquito borne disease common in tropical and sub-tropical countries including Malaysia. There is at present a lack of specific treatment and an effective tetravalent vaccine against dengue. The control of dengue depends solely on the suppression of the two most important vectors namely, Aedes aegypti and Ae albopictus. Despite intensive and extensive control efforts by health agencies, the disease continues to spread. This paper updates various innovations on control of dengue vectors. Gene-based sterile insect technique using the RIDL technology for both Aedes aegypti \& Ae albopictus control has now been actively researched and field trials are pursued to evaluate the effectiveness of the technology. The release of Wolbachia-infected Ae aegypti is another dengue control innovation. The infected mosquito cannot support development of dengue virus and has shorter life span. Other innovations include: auto-dissemination of insect control agents using ovitrap, autocidal adult and larva trap, outdoor residual spraying, insecticidal paint and biocontrol agent. In other innovation, outbreak prediction capability is enhanced by developing model based on environmental data and analysis utilising neural network.
\end{abstract}

KEYWORDS: Dengue, vector-borne disease, mosquito, aedes aegypti, update

\section{INTRODUCTION}

Dengue is the most rapidly spreading vector borne disease in the world. ${ }^{1}$ About $55 \%$ of the world's population (3.6 billion people in 124 countries) is at risk, with over 100 million cases annually. ${ }^{2}$ Malaysia is one of the worst affected countries, with cases recorded increased from 7,103 cases in 2000 to 108,698 in 2014, with 215 deaths, and not less than 31,500 notified cases in any given year. The virus that causes dengue and dengue hemorrhagic fever is an arbovirus with four antigenically distinct serotypes (DEN-1, 2, 3 and 4) that offer no long-term cross-protective immunity against each other. All four serotypes are endemic in Malaysia and South East Asia; however, the predominant circulating serotype is dependent on the herd immunity. Unfortunately, there is no specific treatment for dengue, and a safe tetravalent vaccine is still in

\section{Corresponding author:}

Dr. Lee Han Lim,

Head,

Division of Entomology

Institute for Medical Research,

Jalan Pahang,

50588 Kuala Lumpur,

Malaysia.

Email: leehl@imr.gov.my development and at least a decade away from being available for widespread use. ${ }^{1,3}$ As Aedes is a day-biting mosquito, treated bed nets are also not very effective against Aedes-borne diseases like dengue or chikungunya.

The current strategy is to control the mosquito vectors, Aedes aegypti (L.) (primary vector) and Aedes albopictus Skuse (secondary vector). Mosquito control in a dengue programme generally comprises 3 major components: Vector control \& surveillance; community participation and enforcement. In vector control, application of insecticides is often used widely to reduce and kill the infected mosquito population whenever cases are reported. The application of insecticides especially the pyrethroids is effected through space application in the form of cold or thermal fogging. The main purpose of space spraying is to kill the infected mosquitoes thereby interrupting the transmission cycle \& stop the disease cycle, hence stopping the spread and occurrence of dengue. Surveillance is generally done through house-to-house larval survey and the results are used to formulate several indicators, most commonly the House Index and Breteau Index. These indices are used to denote the risk areas. In most countries, larval survey is used as a form of enforcement, rather than as indicators of Aedes breeding. Thus most of the time, larval indices are not reflective of the true \& actual situation of larval population in the field, rendering $\mathrm{HI}$ \& $\mathrm{BI}$ inaccurate as indicators of breeding.

However, these methods cannot prevent dengue outbreaks by themselves as seen from examples around 
the world, especially Singapore. ${ }^{4}$ A key reason for this is the low threshold for dengue transmission; as low as 2-3 adult female mosquitoes emerging every day in a locality of 100 people. ${ }^{5}$

This does not call for the discontinuation of conventional vector control methods; in fact it would be necessary to continue public education and 'COMmunication for Behavioural Impact' (COMBI) for sustained breeding site reduction involving the public. Legally-enforced larval control, whether by breeding site elimination or larviciding, is neither practical for a large and diverse country like Malaysia, nor has it stopped the resurgence of dengue in Singapore. ${ }^{4}$ Aedes aegypti breeds in a diverse set of small containers, often rainwater-filled, therefore finding and treating all or most of these containers are impractical. However, none of the aforesaid methods, by themselves or together, have been able to stop the spread of dengue, mainly due to not being able to reduce the mosquito population below the low transmission threshold, as mentioned above.

\section{RECENT ADVANCES IN DENGUE CONTROL}

New method, novel ideas and concepts are urgently needed to revamp the whole dengue control strategies and to check the spread of dengue which apparently at present is unstoppable. We have therefore refocused our research efforts on the development and evaluation of new tools aiming at reducing the vector population below the low dengue transmission threshold.

\section{Insecticide-based}

\section{(i) Outdoor residual spraying (ORS)}

Residual insecticide indoor and outdoor spraying is a commonly used technique for obtaining a reduction of vector man contact. This technique is applied to control the malaria vector and is still an applicable control method in tropical countries. In Malaysia, the first experiment on Ae. aegypti control was carried out at Port Swettenham in $1952 .{ }^{6}$ After a preliminary survey by Reid (1954), residual spray with DDT was carried out. ${ }^{7}$ However the results were not very encouraging. Another experiment was conducted in Jeram village and found to give almost complete control of Ae. aegypti for a year or more. ${ }^{8}$ Rozilawati et al (2005) showed that indoor and outdoor residual spraying was effective in controlling the Aedes mosquito especially in the area where the population was high and the preventive maintenance could not be implemented. ${ }^{9}$

Recently, we conducted a field study to evaluate the effectiveness of outdoor wall residual spraying in high rise building for the control of dengue. A new formulation of pyrethroid (Suspend $®$ Polyzon, Bayer) was field tested in this study. The formulation, which contains deltamethrin-coated polymer, was applied at $30 \mathrm{mg} / \mathrm{m}^{2}$ on the outer wall surface of each residential unit in a five storey apartment. Two cycles of spraying lasting 8 weeks each were conducted and evaluated for one year. Another similar apartment not treated with residual spraying but subjected to conventional dengue control activities was used as a control. The effectiveness of the residual spray was evaluated by the impact on vector population and diseases incidence using ovitrapping, WHO wall deposit bioassay and dengue epidemiological data .The results showed that the ovitrap index in treated area was lower ( $\leq 50 \%$ less) compared to the control sites one month after spray. Wall deposit bioassay showed that the knockdown rate for Aedes aegypti tested indoor was $>50 \%$ for eight weeks and the $24 \mathrm{~h}$ postmortality was> $80 \%$ for 12 weeks. Aedes albopictus showed lower knockdown rate $(45 \%$ - $80 \%)$ but the mortality was higher (> 80\%). However, outdoor knockdown and mortality rate were variable, $20 \%$ $100 \%$ for knockdown and 30\%-100\% for mortality. The insecticide residual was most effective one month after spray and reduced thereafter. No dengue cases were reported in treated area during the study. Environmental factors such as sunlight and rainfall affected the knockdown and mortality of the mosquito. Outdoor residual spraying of high rise building is an effective method in controlling dengue. ${ }^{10}$

\section{(ii) Autodissemination trap}

Insect Growth Regulator (IGR)-treated ovitraps have been shown promising in reducing $A e$. aegypti populations. An IGR, pyriproxifen is now used in autodissemination trap to control Aedes. This is a large ovitrap containing about $250 \mathrm{ml}$ water and $0.004 \%$ pyriproxifen. Female mosquitoes attracted to lay eggs into the trap will contaminate themselves with the IGR and when they visit \& lay eggs into another container, pyriproxifen is transferred into the water \& affects the larvae that hatch subsequently. This is feasible as pyriproxifen acts at very low dosage.

We conducted field trial of a pyriproxifen treated autodisemination trap in 3 blocks of residential condominium in Selangor. Each block consists of 27 storeys with 10 units in each floor. Each floor is approximately 7,500 $\mathrm{ft}^{2}$. Breeding of Aedes aegypti has been found on every floor until the $27^{\text {th }}$ floor. Four traps were used per level in each \& every block and a total of 350 traps were deployed. The placement of each device was in the corridor at an interval distance of 5 meters outside each apartment unit. The trial was conducted for almost a year.

The solution of the autodissemination trap seemed to have an attractant component which induced the females to lay eggs. Although the eggs in autodissemination traps hatched, no single surviving larva was observed in all the traps placed with the presence of hatched and unhatched eggs, showing $100 \%$ mortality throughout the 45 weeks of trials (10 months). The ovitrap index declined and more importantly, significant impact of auto-dissemination trap on suppressing dengue transmission was observed and apparently interrupted with 53 confirmed dengue cases reported in 2013 prior to the trial, reduced to 13 cases at the end of the trial in 2014. ${ }^{11}$ 


\section{(iii) Insecticide with both adulticiding \& larviciding property}

The organophosphate temephos is traditionally used as a larvicide for the control of pests and insects of public health importance. Two commercial formulations are available; Abate 1-SG ${ }^{\mathrm{TM}}$ which is a granular formulation and Abate $500 \mathrm{EC}^{\mathrm{TM}}$, an emusifiable concentrate formulation. Abate $500 \mathrm{EC}^{\mathrm{TM}}$ has been used traditionally as a larvicide to control mosquito larvae. Studies conducted in IMR in 1999 indicated that Abate $500 \mathrm{EC}^{\mathrm{TM}}$ applied at $200 \mathrm{~g}$ a.i./ ha also exhibited both adulticidal \& larvicidal effects on Aedes aegypti and housefly. In this present study, the effectiveness of space-sprayed Abate $500 \mathrm{EC}^{\mathrm{TM}}$ in the control of dengue and the vectors was assessed in a 7 ha housing estate. A similar area which is $>1 \mathrm{~km}$ away was used as an untreated control. In the first study, lab-bred Ae aegypti in cages, empty paper cups and magnesium oxide coated glass slides were placed indoor \& outdoor of houses. Abate 500EC ${ }^{\mathrm{TM}}$ was applied at $200 \mathrm{~g}$ a.i./ha using an truck-mounted ULV generator. Complete adult mortality and significant larval mortality was observed $24 \mathrm{~h}$ post-spraying. Subsequently, the trial site was sprayed weekly with Abate $500 \mathrm{EC}^{\mathrm{TM}}$ at $200 \mathrm{~g}$ a.i./ ha. Two thermal foggers were also used. Area treated with Abate $500 \mathrm{EC}^{\mathrm{T}}$ generally showed lower Aedes population as indicated by the ovitrap index. The application of Abate 500EC ${ }^{\text {TM }}$ also interrupted dengue transmission in the treated site. It is concluded that space-sprayed Abate $500 \mathrm{EC}^{\mathrm{TM}}$ at $200 \mathrm{~g}$ a.i./ha is effective in Aedes and dengue control. ${ }^{12}$

\section{(iv) Insecticidal emulsion paint}

The insecticidal paint is an emulsion paint formulation impregnated with an insecticide and is developed as a simple but very effective means of delivering a chemical insecticide for the control and elimination of insect pests, especially those in houses, food-processing and production factories, hospitals, ships etc. where the usual way of insecticide application is inappropriate or hazardous. Since painting is widely practised in many activities, this product will ensure its widespread use which will result in effective insect control and elimination. The insecticidal paint contains $0.25 \%$ (active ingredient, $v / v$ ) of a synthetic pyrethroid, deltamethrin and $0.25 \%$ of a synergist, piperonyl butoxide. ${ }^{13}$ Deltamethrin is used widely as a residual wall spray since 1998 for the control of malaria in Malaysia and other countries. It is also used in bednet impregnation used for protection against malaria vectors. Piperonyl butoxide is included to counter or delay the possible development of resistance in insects.

The efficacy of the paint formulation was tested extensively in IMR laboratory against all major insects. A normal painting of the insecticidal paint on wood or cement surface induced high mortality in the test mosquito adults of Aedes aegypti, Aedes albopictus, Anopheles maculatus and Culex quinquefasciatus, and in the housefly Musca domestica. The initial killing effect on the Greman cockroach, Blattella germanica was generally low but increased to high mortality after several weeks. The killing effects lasted more than 2 years in laboratory tests. In the field, a small scale test in a small kitchen (14' $\left.\times 7^{\prime}\right)$ resulted in 3 years control of cockroaches, housefly, ants and lizards. The kitchen was pest-free for almost 3 years. Field testing of the insecticidal paint against dengue will be conducted in the near future.

\section{Biological-based}

\section{(i) Wolbachia}

Wolbachia were first identified in the ovaries of Culex mosquitoes in 1924. Wolbachia species are obligate intracellular rickettsia-like bacteria belonging to the alpha subclass of Proteobacteria and the order of Rickettsiales that live inside the cells of various organs, but most frequently appear in ovaries and testes. Wolbachia infects a wide range of arthropods including mosquitoes, ticks, flies and nematodes populations. However, some of the major disease vectors are not naturally infected, including the primary vector of dengue, Ae. aegypti and all anopheline mosquitoes sampled to date. The bacteria are maternally inherited through host eggs and reaching their optimum fitness by altering the reproduction of their host via several mechanisms including parthenogenesis, feminization, malekilling and cytoplasmic incompatibility $(\mathrm{Cl})$. Among all the reproductive modifications caused by Wolbachia, $\mathrm{Cl}$ has been utilized as one of the tools to fight against mosquitoes. $\mathrm{Cl}$ is a form of sterility in which if the same and compatible Wolbachia strain is not present in the egg during embryogenesis, embryonic development will be disrupted. Interestingly, some Wolbachia strains are able to cause pathogen interference including viruses in their newly introduced invertebrate hosts and yet, conferring no effects on their original host. For example, the introduction of Wolbachia strain B isolated from Ae. albopictus (wAlbB) into a novel host, Ae. aegypti via embryonic injections of the eggs has shown to induce dengue resistance. A decrease in the viral proliferation of Chikungunya virus (CHIKV) was also observed in wMelPop infected Ae. aegypti. ${ }^{14}$

Prior to utilizing Wolbachia as novel vector control strategies, it is crucial to understand the Wolbachia mosquito interactions. In our study, surveys were conducted on field-collected Aedes albopictus from various habitats ranging from housing areas, islands and seashore. A total of 156 pools of mosquitoes were analyzed using multiplex polymerase chain reaction (PCR) and gene sequencing. The results indicates that these bacteria are widespread with 73/76 (female) and 83/87 (male) were positive with Wolbachia infection. The wsp gene sequence of the Wolbachia strain from 104 individual mosquitoes was BLAST and showed a 100\% homology with Wolbachia sp. of Aedes albopictus isolated from various geographical regions. This is the first report of the presence of Wolbachia from mosquitoes in Malaysia. ${ }^{15}$ 


\section{(ii) Bacillus thuringiensis $\mathrm{H}-14$}

Bacillus thuringiensis israelensis (Bti) is a microbial control agent that effectively kills the larval stage of mosquitoes such as Aedes aegypti and Aedes albopictus without undesirable effects on the environment and non-target organisms. After Bti ingestion, the alkaline $\mathrm{pH}$ environment in the larvae intestine will activate the degradation of the Bti endotoxin into mioety which is highly toxic to the mosquito larvae. ${ }^{16}$ We conducted an operational application of Bti to control dengue. In this study, the effectiveness of a space-sprayed Bti formulation, VectoBac WG ${ }^{\circledR}$ (3000 ITU/mg) in the control of dengue was assessed in two dengue hotspot areas using truck-mounted Ultra Low Volume (ULV) generator and mist blower. The application dosage was $400 \mathrm{~g} / \mathrm{ha}$ for ULV generator, while for mist-blower, the formulation was prepared at $600 \mathrm{~g}$ in 10 litres of water. The latter was used to cover areas that were not reachable by ULV. The spray was conducted weekly. A significant reduction in the number of dengue cases was observed after the third Bti application for both areas. After this, the number of dengue cases continued to drop during treatment period in both sites until no cases were reported. Eventhough there was a slight increase in the number of dengue cases observed post-treatment, the number of cases was lower compared to pre-treatment period and these areas were no longer declared as dengue hotpsots. The ovitrap index (OI) in both treated site continued to fluctuate during the treatment period, most probably due to the migration of mosquitoes from neighbouring areas. Space sprayed $B t i$ is an effective larvicidiing agent against Aedes mosquitoes and dengue, and this method is applicable in mass treatment of dengue outbreak areas (Nazni et al, 2014, unpublished).

\section{Gene-based}

\section{(i) Genetically-modified Aedes aegypti}

Aedes aegypti is genetically modified using a technology known as RIDL (Release of Insects carrying Dominant Letha). The science behind RIDL is known as 'repressible lethality', i.e. introduction of a specific DNA construct into Aedes aegypti eggs through micro-injection so that the transformed mosquito is destined to die at larval or pupal stage unless it is provided with a nutritional supplement (tetracycline) in the rearing medium. This supplement represses the lethal gene and hence allows the mosquito larvae to grow normally into adults when they are reared in a laboratory or rearing facility. However, these larvae are destined to die in the wild due to the absence of tetracycline, which is an unstable compound in the environment, degrading by several routes including photo-degradation in sunlight with a half-life under two hours at $\mathrm{pH}$ 8. By releasing large numbers of these sterile male mosquitoes (male mosquitoes cannot bite) in a sustained manner, Aedes aegypti populationcan becrashed below the diseasetransmission threshold, and possibly even eradicated within a year fromlargecommunities. TheRIDLstrainwasimportedand successfully colonised in purpose-built facilities at the IMR. Many physical and biological traits of RIDL strain were measured, relating to all life cycle stages from egg to adult. ${ }^{17-22}$ No significant differences were detected between the RIDL strain and a control wild type strain. The comprehensive tests indicated that RIDL has promise as a new vector control tool, without any adverse effects observed. Male mating competitiveness experiments were also conducted in a purpose-built field house at the IMR for the first time. Male mating competitiveness, a key determinant of success in a sterile male release program, was carefully measured under conditions that mimic the natural domestic and peridomestic environment of the mosquito. The RIDL strain performed extremely well; male mating competitiveness was equivalent to that of the control unmodified strain. ${ }^{23}$ Mark-Release-Recapture \& field release of RIDLmosquitoexperimentwasconductedafter extensive regulatory approval in an uninhabited area. The results showed that the flight distance and longevity of the RIDL male mosquito was similar to the wild untransformed type. ${ }^{24}$ Based on these studies, the first suppression trial using RIDL $A e$ aegypti was conducted in Cayman Islands and achieved $82 \%$ suppression of the population. Subsequently, sustained released of RIDL Ae aegypti in 3 areas in Brazil also achieved $81 \%, 91 \%$ and $95 \%$ suppression of $A e$ aegypti.

\section{(ii) Sterile Insect Technique}

The idea of releasing sterile insects in large numbers to control pests is itself not new, but a wellknown concept that was developed in the 1950s. The Sterile Insect Technique (SIT) has set very successful precedents around the world, most notably by controlling the Mediterranean fruit fly or medfly (Ceratitis capitata), and by eradicating the new world screw worm (Cochliomyia hominivorax) from the United States and Central America all the way down to Panama. However, classical SIT has hitherto not been successful with mosquitoes such as Aedes in spite of much effort because gamma radiation, used in classical SIT to sterilise the insects, renders the mosquitoes very weak and unfit to compete with the wild male mosquitoes. Hence studies are now focusing on finding the optimal radiation dosage that ensures complete male sterility while preserving mating competitiveness of sterile males. We conducted a study aimed at determining the optimum sterilizing dose rate of Cesium-137 gamma irradiation and impact on life stage parameters of gamma rayirradiated wild-type and laboratory strain of Aedes aegypti male. Male pupae of each strain were exposed to six different dosages ( $5 \mathrm{~Gy}$ - $100 \mathrm{~Gy}$ ) of ${ }^{137} \mathrm{Cs}$ gamma irradiation. The results indicated that the effective irradiation dosage inducing complete male sterility was 45 Gy to 80 Gy. No significant difference was observed in adult emergence and mortality in both strains $(p>0.05)$. However, laboratory reared male mosquitoes were more susceptible to irradiation especially at higher doses resulting in decreased life span. The presence of 
sperms in female spermathecae showed that the mating ability of the irradiated males was not impaired. ${ }^{25}$ These promising results warrant further study especially field release possibility.

\section{Mechanical-based}

\section{(i) Autocidal trap}

A new autocidal trap known as IMR Autocidal Trap (IAT) device was first designed by us. It is a modification of the traditional ovitrap and is able to trap both the gravid female mosquitoes and the larvae. The patented IAT is cost-effective, environmentalfriendly, user-friendly, durable and safe to use and with minimum maintenance. An important component of the trap is a sticky plastic strip attached on top of the floater. The sticky surface can trap gravid mosquitoes that are attracted to water in the black container for oviposition. The trap was field tested in 2 residential areas whereby 85 houses were selected and each was installed with three IATs placed indoor (2) and outdoor (1). The sticky strips were collected biweekly and the insects trapped on the strip were identified, counted and recorded. Female Ae. aegypti was the predominant mosquito trapped in IAT(42.8\%), followed by female $C x$. quinquefasciatus (17.9\%) and $5.56 \%$ of female Ae. albopictus. A mean of 2 Aedes mosquitoes were trapped/strip/2 weeks / house. On the average, about $21 \%$ of the participating houses successfully trapped Aedes specie during each visit. Eggs were also laid on the floaters, showing that gravid female mosquitoes were attracted to oviposit in the IATs. ${ }^{10}$ Subsequently, a large scale trial was conducted in Shah Alam, comprising 3000 units of apartment, terraced houses and semi-detached houses. Each of the apartment was installed with 2 IATs, while the terraced houses and semi-detached houses were each installed with 3 IATs and 4 IATs respectively. Preliminary results indicated that Aedes and other adult mosquitoes were trapped and the ovitrap index was reduced in treated premises. The impact on dengue transmission was evident from the decreasing trend of cases reported.

\section{Environmental-based}

\section{(i) Dengue outbreak prediction model}

Early detection of a dengue outbreak is an important first step towards implementing effective dengue interventions resulting in reduced mortality and morbidity. A dengue mathematical model would be useful for the prediction of outbreak and evaluation of control measure. However, such model must be carefully parameterized and validated with epidemiological, ecological and entomological data. Multiple intrinsic factors, both human and vector, and extrinsic environmental factors such as temperature, rainfall and relative humidity affect epidemic dengue virus transmission. ${ }^{26}$ In a preliminary study, localized rainfall data and dengue cases from a residential area in Kuala Lumpur were analysed using a software (called Engine) based on Artificial Intelligence mimicking human neuron (Neural Network
- NN). The software is able to process and analyse voluminous data and also able to "learn" by "training" which means the software is able to self-improve when new data is provided. Initial testing of the model indicated a promising prediction accuracy of $65 \%$ three weeks in advance of the outbreak (Rohani, 2014, unpublished). However, obviously the accuracy needs to be further enhanced and large scale field testing should be conducted to verify the model's feasibility to provide advanced warning and hence early remedial actions taken to avert the outbreak.

\section{CONCLUSION}

After decades of limited success in controlling dengue $\&$ its vectors, it is now abundantly clear that present dengue vector control technology is inadequate and this calls for urgent development of new and effective control tools to address the seemingly unstoppable dengue problem. However, any new tool developed cannot be applied stand alone. To ensure maximal and optimal effectiveness, a multi-pronged approach integrating these new tools is a far more effective mean of control. For instance, outdoor residual spraying can be applied in conjunction with autocidal trap or autodissemination trap; Wolbachia-infected Ae aegypti can be released into areas treated with ORS or insecticidal paint; area-wide mass application of Bti to reduce natural Ae aegypti population prior to release of RIDL male mosquitoes etc. Future research on dengue control should therefore be focused on finding the optimal integration of new tools, the possibility of scaling up and translation into control programmes and cost-effectiveness analysis.

\section{Acknowledgements}

The authors thank the Director General of Health, Malaysia for permission to publish, and Director of Institute for Medical Research, Kuala Lumpur for support.

\section{REFERENCES}

1. World Health Organization, Report of the Scientific Working Group meeting on Dengue, Geneva, Switzerland, 1-5 October 2006.

2. Beatty ME, et al. International Vaccine Institute, USA. Estimating the total world population at risk for locally acquired dengue infection. 56th Annual Meeting of the American Society of Tropical Medicine and Hygiene, Philadelphia, USA, 4-8 November 2007.

3. World Health Organization Fact Sheet No 117: Dengue and dengue hemorrhagic fever, Geneva, Switzerland, April 2002.

4. Curtis C. Review of previous applications of genetics to vector control. In: Knols BGJ, Louis $C$, eds. Bridging Laboratory and Field Research for Genetic Control of Disease Vectors. Netherlands: Springer, 2006. 
5. Focks DA, Brenner RJ, Hayes J, Daniels E. Transmission thresholds for dengue in terms of Aedes aegypti pupae per person with discussion of their utility in source reduction efforts. Am J Trop Med Hyg 2000; 62:11-18.

6. Cheong WH. The vectors of dengue and dengue haemorrhagic fever in Malaysia. In: Rudnick A, Lim TW, eds. Dengue fever studies in Malaysia, Bulletin 23. Kuala Lumpur, Malaysia: Institute of Medical Research, 1986: 155-167.

7. Reid JA. A preliminary Aedes aegypti survey. Med J Malaya 1954; 9:161-8.

8. Macdonald WM. Aedes aegypti in Malaya IIIexperiments on control and eradication. Ann Trop Med Parasit 1957; 5:175-86.

9. Rozilawati H, Lee HL, Mohd-Masri S, Mohd-Noor I, Rosman S. Field bioefficacy of delthamethrin residual spraying against dengue vectors. Trop Biomed 2005; 22:143-8.

10. Nurulhusna AH, Rosilawati R, Mohd lqbal MN, et al. Outdoor residual spraying of a novel deltamethrin formulation for the control of dengue in high rise building. Paper presented at 51st Annual Scientific Seminar of the Malaysian Society of Parasitology \& Tropical Medicine: Kuala Lumpur, 3-4 March, 2015.

11. Nazni WA, Oreenaiza N, Teoh GN, et al. Field evaluation of auto-dissemination traps for the control of dengue in high-rise condominium in Selangor. Paper presented at 51st Annual Scientific Seminar of the Malaysian Society of Parasitology \& Tropical Medicine: Kuala Lumpur, 3-4 March, 2015.

12. Nazni WA, Teoh GN, Rosilawati R, et al. ULV application of temephos (Abate ${ }^{\circ} 500 \mathrm{E}$ ) for the control of dengue. Paper presented at 51st Annual Scientific Seminar of the Malaysian Society of Parasitology \& Tropical Medicine: Kuala Lumpur, 3-4 March, 2015.

13. Lee HL, Nazni WA, Abdullah AG. Insecticidal paint composition. Patent No. MY-132793, Granted 31 Oct 2007, MyIPO.

14. Noor Afizah, A, Lee HL. Wolbachia-based control strategy for dengue control. Dengue Bulletin 2014; 37:107-15.

15. Noor Afizah A, Roziah A, Nazni WA, Lee HL.Detection of the endosymbiont Wolbachia from field-collected Aedes albopictus Skuse in Malaysia. Indian J Med Res (in press)

16. Lee HL. Germ warfare against mosquitoeswhat now? Proceedings of the International Conference on Urban Pests: Singapore, 11-13 Jul, 2005.

17. Lee HL, Aramu M, Nazni WA, Selvi S, Seshadri $V$. No evidence of interspecific cross-mating of transgenic Aedes aegypti (L) and wild type Aedes albopictus Skuse. Trop Biomed 2009; 26:312-9.

18. Lee HL, Joko H, Nazni WA, Seshadri V. Comparative life parameters of transgenic and wild strain of Aedes aegypti (L.) in the laboratory. Dengue Bulletin 2009; 33:103-14.

19. Nazni WA, Selvi S, Lee HL, et al.
Susceptibility status of transgenic Aedes aegypti (L.) against insecticides. Dengue Bulletin 2009; 33:124-9.

20. Nordin MO, Donald W, Ming HW, et al. Oral ingestion of transgenic RIDL Ae. aegypti larvae has no negative effect on two predator Toxorhynchites species. PLoS ONE 2013; 8: e58805.

21. Angamuthu C, Haninah A, Renaud L, et al. Similar susceptibility of transgenic and nontransformed Aedes aegypti (L.) to dengue and chikungunya. Transgenic Research (in press)

22. Suria Marlina M, Haninah AUl, Renaud L, et al. Similar transovarial transmission of dengue and chikungunya viruses in transgenic and non-transformed Aedes aegypti (L.). Trop Biomed (in press).

23. Lee HL, Seshadri V, Nazni WA, et al. Mating compatibility and competitiveness of transgenic and wild type Aedes aegypti (L.) under contained semi-field conditions. Transgenic Res 2013; 22:47-57.

24. Lacroix R, McKemey AR, Raduan N, et al. Open Field Release of Genetically Engineered Sterile Male Aedes aegypti in Malaysia. PLoS One 2012; 7: e42771.

25. Nazni, WA, Teoh GN, Apeksha LW, et al. Life stage parameters of Cesium-137 irradiated and normal wild-type and laboratory strain of Aedes aegypti male. Paper presented at 51st Annual Scientific Seminar of the Malaysian Society of Parasitology \& Tropical Medicine: Kuala Lumpur, 3-4 March, 2015.

26. Rohani A, Suzilah I, Malinda M, et al. Aedes larval population dyanamics and risk for dengue epidemics in Malaysia. Trop Biomed 2011; 28:237-48. 